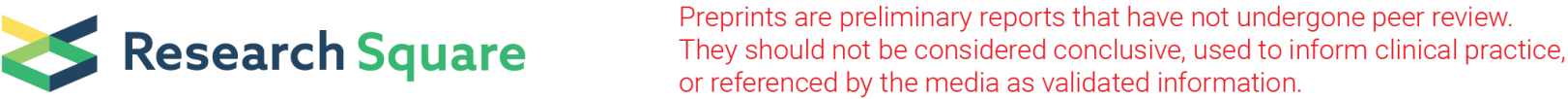

\section{Multi-dimensional strategic approach to the communicable diseases with systemic risks by using IT2 fuzzy decision-making modelling}

\section{Shubin Wang}

Xi'an University of Arts and Science

Peiqing Yang ( $\nabla$ ypq@xupt.edu.cn )

Xi'an University of Arts and Science

Serhat Yüksel ( $\nabla$ serhatyuksel@medipol.edu.tr)

Istanbul Medipol University https://orcid.org/0000-0002-9858-1266

Hasan Dinçer

Istanbul Medipol Universitesi

Gözde Gülseven Ubay

Istanbul Medipol Universitesi

\section{Research}

Keywords: Communicable Diseases, Socio-economic Burden, Strategy, Systemic Risks, IVIF DEMATEL

Posted Date: July 23rd, 2020

DOI: https://doi.org/10.21203/rs.3.rs-39557/v1

License: (c) (1) This work is licensed under a Creative Commons Attribution 4.0 International License. Read Full License 


\section{Abstract}

Background: Communicable diseases lead to significant social and financial burden for the countries. Therefore, countries should define their social and technical factors for struggling these problems. The main purpose of this study is to determine the effective strategies to minimize socio-economic burden of these diseases.

Methods: For this purpose, 8 different criteria are identified based on the literature analysis. In the evaluation process, the significance levels of these factors are calculated by considering interval-valued intuitionistic fuzzy (IVIF) DEMATEL.

Results: The findings show that technological infrastructure, social distance, and tendency of obeying rules are the most significant items to control these problems. This situation explains that countries should have the necessary equipment to make the diagnosis quickly and effectively. In addition, countries that have effective laboratories in which vaccines and drugs can be developed can be more successful in combating these pandemics. Additionally, it is also defined that cultural factors play also key role to control these problems.

Conclusions: In this framework, it is vital that the people in the country take the rules set by the government seriously and without exception. For this purpose, it should be explained to citizens living in the country that personal hygiene, social distance and not entering crowded environments are very important to manage these problems. For this purpose, role model people in the country can be preferred in different platforms so that it can be more effective on the citizens.

\section{Introduction}

Accelerated spread of a disease to a high amount of people in each population within a short period of time is called communicable diseases. If these diseases have destructive effects, they are called as pandemics. [1]. The probability of pandemics has raised over the past century as a result of the disappearance of borders due to globalization, urbanization, changes in land use, and larger exploitation of the natural atmosphere. At this point, COVID-19, one of the new China-based corona viruses that have appeared in the last months and spread to the world in a brief time, can be given as an example. All those pandemics poses a great danger to many people and animals and is a threat for ecological balance of the world. Societies that infected with pandemics use many ways to prevent the spread of such diseases and to get rid of them. The main reason is that most of the time, the number of people who died due to pandemic diseases can be more than the number of people who died due to war or natural disasters. Besides the threatening dimensions of the pandemics to individuals and animals, it is observed that the pandemics have also serious economic, social and cultural consequences on the countries [2].

While many studies unite that outbreaks will have a negative impact on national economies and the global economy, the cost of these pandemics may worsen if the number of people infected or mortality increases [3]. When the infection rate increases to a significant level, governments' health spending also 
must increase to control this problem. This situation creates a financial burden on countries' budgets. On the other hand, there is always a lack of information about how these pandemic diseases spread and how bad they can be. This contributes to global uncertainty in the process and increases the concerns of companies and investors. In general, pandemic diseases that have emerged since 1970 have been affecting the global markets negatively over a period. As a result, there is a concern that economic growth will be limited due to the spread of the viruses [4].

In another aspect, it is obvious that pandemics lead to systemic risks for many countries. In other words, pandemics negatively affect companies in different sectors with their contamination effect. As a result, these companies are subject to serious losses. Due to the systemic risk created by the pandemic, companies face liquidity risk. This situation causes companies to fail to pay their short-term debts. After the emergence of systemic risk, the uncertainty in the market increases and this causes investors to be nervous. This problem leads to increased vulnerability in both financial markets and macroeconomics. Within this framework, in countries where there is a risk of spreading of these types of pandemic diseases, tourism, transportation, trade and services may be adversely affected due to lower customer demands [5].

Besides economic results, there is also a social aspect for pandemics. Protective and preventive applications taken in pandemics may have some social consequences. The social impacts of pandemics can be catastrophic. It can cause considerable losses in the total population and this can lead to fragmentation of families or societies. Furthermore, many pandemic diseases can have chronic effects in humans' life such as panic attack due to fear, which can become more common in time. In the other hand, as it is seen in COVID-19 case, pandemics can have important social consequences, creating conflicts between states and citizens, harming state capacity, eroding social cohesion, and raising social tension and discrimination [6]. It is very clear that pandemics have serious impacts on both economic and social aspects of life. For countries, in order to get affected as less as possible from these negativities, emphasis should be placed on factors that strengthen the fight against pandemics.

In today's global society, pandemic disease outbreaks can spread quickly all around the world. At this point, improving health sector can be very beneficial for countries to prevent pandemics to spread or to control after it is spread in the country. Taking into action certain activities may be very beneficial before they become a wider threat [7]. The infrastructure of a country's health sector is at the core of pandemic prevention. Countries with improved health infrastructure can react more easily to a potential pandemic. Since such countries will have sufficient equipment and qualified health personnel, it becomes easier for them to control an outbreak in their country. In the other hand, countries with high technological infrastructure also get successful in the process of managing pandemics. The technological infrastructure in the health sector helps to make the tests on the pandemics more easily and at regular intervals, a better examination process with advanced technology equipment and to find the cure for some pandemics earlier and easier. 
In the other aspect, population planning is one of the important points. For example, in the regions where the population is dense, the human interaction is also intense. Because of this situation, it can be said that they are at higher risk of pandemic diseases [8]. For this reason, good population planning makes an important contribution to manage the pandemic disease prevention process. Besides population planning, people's awareness towards social distance can be crucial at this point. Some societies choose a closer communication method among themselves, while others may be more distant. The cultural structure of the society in a country can shape the spread of the outbreak. When there is a destruction of social distance, it will be harder for countries to get success in pandemics. To manage this process successfully, some necessary steps have to be taken into account by countries.

As mentioned in general, producing specific strategies on the topic of pandemics is of great importance for both people and countries. For this reason, it will be very useful to create an order of importance when creating these strategies. The method to be used in this context is also of great importance. Econometric methods can be very helpful to make necessary prediction for the future of the problem. However, the main drawback of these approaches is that non-numerical data cannot be considered in the analysis process [9]. For this reason, the appropriateness of these methods is questioned [10]. Therefore, it would be more accurate to use different methods that also include non-numerical data to provide more appropriate recommendations. At this point, multi-criteria decision-making methods (MCDM) make it possible to examine both numerical and non-numerical data. For this reason, these methods have been used quite frequently in the literature especially in recent years within the framework of fuzzy logic. Thus, the complexities and uncertainties in the decision-making process can be reduced [11].

The aim of this study is to investigate which factors are more effective in strengthening countries' struggle with pandemics. In this context, as a first step, with the help of literature scanning 8 different factors that can have impact on that managing process are identified in two dimensions. Later, an analyze is conducted with IVIF DEMATEL method to determine the order of importance of factors. This approach has many advantages in comparison with the similar ones. For instance, the causality relationship can be conducted with this method because impact-relation map can be generated $[12,13]$. On the other side, uncertainty and ambiguity in decision-making process can be coped with more effectively with the help IVIF methodology [14].

At this point, many different advantages of this study can be mentioned. As a first, by presenting the weighted factors, it will be possible to identify which factors are more important to manage pandemics for countries. This can help academicians in their future works and can be very beneficial for countries to take appropriate precautions. This may also contribute to the reduction of effects of pandemics on countries. Since COVID-19 is most recent and severe virus, it will be possible to offer suggestions for these countries to better manage the process that are still going on and pave a way for preventing possible pandemic threats. Another important contribution of the study to the literature is methods that are used in the analysis process. IVIF DEMATEL method is considered for the first time in this study in the framework of the pandemics. It is thought that this situation increases the methodological novelty of the study. 
This study contains 6 different sections. In the first section of the study, a general information about pandemics and their effects on social and economic areas are shared. Later, in the second part of the study, a brief history of COVID-19 and updated information are given. Furthermore, in the third section of the study, a literature review is conducted on factors that affect success of a country for managing pandemics. In addition, the fourth section of the study covers the theoretical knowledge of IVIF DEMATEL approach used in the analysis process. Moreover, in the fifth part of the study, the results of the analysis are covered. And finally, the sixth part of the study contains the strategy recommendations that developed according to the analysis results obtained.

\section{General Information On Covid-19}

Corona viruses (CoV) can cause different diseases, from mild colds to severe respiratory distress such as the Middle East Respiratory Syndrome (MERS-CoV) and Severe Acute Respiratory Syndrome (SARS-CoV). A novel type of coronaviruses, COVID-19 is a virus identified on January 13,2020 , as a result of investigations in a group of patients who developed fever, cough, and shortness of breath in Wuhan Province in late December, China. The pandemic was initially detected in those in the seafood and animal market in this region [15]. Then it spread from person to person and spread to other cities in the province of Hubei, mainly Wuhan, and other areas of the People's Republic of China and finally other countries of the world. Because it is transmitted by inhaling droplets that are scattered by sick coughing and sneezing, unfortunately the spread speed of COVID-19 is quite fast.

According to the information obtained so far about COVID-19 infection, it is concluded that some people are at higher risk of getting sick and developing serious symptoms. The disease usually affects people aged 60 and over more and people that have chronic medical conditions, heart disease, and so on [16]. In addition, health workers are also at high risk, since this virus can be transmitted very quickly. Several efforts to develop vaccines for COVID-19 are underway, but according to World Health Organization it will take 18 months for it to be produced. At present, most of the cure is symptomatic and supportive with the help of anti-inflammatory and antiviral treatments. Unfortunately, specific antiviral drugs that are effective have not been found yet.

The COVID-19 pandemic was recognized as a pandemic by the World Health Organization in early March [17]. Pandemics, seen as a subgroup of epidemics, are a name given to infectious diseases that threaten the health of people living around the world in a period. In the corona virus pandemic that occurred in China, there is a steady increase in deaths and number of cases, while many officials provide information on measures to be taken and the total number of cases. Despite the measures taken by countries, the corona virus has infected thousands of people and has caused lots of people to die. The number of positive cases for this illness has reached 474.204 worldwide. The number of people exposed to COVID19 worldwide is thought to be more than this number. Since the main symptoms of the disease are common symptoms such as fever and cough, many cases have not been examined and considered. 
Apart from China where the virus originated, the most confirmed cases take place in Italy [18]. In this country, the new epicenter of the COVID-19 in Europe, 63.927 cases have been identified to date. 7,503 people died due to this virus. Italy has become the country with the highest mortality rate by passing even China. Spain stands out as the European country where the most cases were seen after Italy. While 35,212 cases have been identified in Spain, 2,318 people have died due to the COVID-19. The US, Germany and Iran also have high amount of confirmed cases. According to the World Health Organization, the US could become the next epicenter of the pandemic due to the rapid spread of cases especially in New York. Whereas new cases cannot be prevented in Europe and the US, China has recently been able to control the number of cases.

As it is mentioned above, the virus, which spread rapidly to the European continent after China, affected many countries, especially Italy and Spain. In this framework, many countries needed to take some actions at both national and international levels. The first of these is to quarantine people who have recently had international contact. With this method, it is aimed to minimize the infectious effect of the virus. Indian government declared a 21-day lockdown across the country recently to support this idea and minimize risks [19]. In addition, curfews have been imposed in several places, many companies have started working from home and many people have decided not to leave the house under the name of selfquarantine. The countries that have most of the cases such as Italy, have imposed strict restrictions, shutting down almost all stores, service and entertain places. Also, education at many schools in these countries has stopped. As a result of the international quarantine incident, restrictions were imposed on most international flights, and some countries even closed their borders completely. Many people in the world are now living under some form of restrictions on their movement and social contact due to the COVID-19.

COVID-19 pandemic has also a negative effect on countries' business and financial conditions. These effects contain disruptions or restrictions on employees' ability to work effectively, as well as temporary closures of companies' facilities. There is a huge reduction of demand in some sectors. Many airline companies make their flights grounded because of the collapse in demand over fears of contagion. This has also affected airports, which have cut hundreds of jobs since coronavirus arrived [20]. Travel bans and airspace closures also have contributed to the temporary suspensions of airlines, ranging from global national carriers to small regional airlines. COVID-19 has also taken a toll on multiple companies which could result in an economic downturn that may negatively affect demand for their products. Small and medium enterprises (SMEs) are one of the most affected company types in this process. It's a hugely uncertain time for them. SMEs business activities has been severely slowed, affecting many countries' position in the global industrial supply chain [21]. With Coronavirus shaking the economies all around the world and forcing many SMEs to close their doors for the foreseeable future, businesses are faced with greater demands for support, including access to finance.

\section{Literature Review}


There is a pandemic of COVID-19 that turns into a global threat at the top of the world agenda. In this context, managing epidemics successfully gains a great importance. This process can be handled in two dimensions. Examining both technical and cultural factors that affect this process is crucial to determine strategies. In literature, there is vast amount of studies in that area. In literature section, firstly, necessary information is given regarding the studies in which factors that helps countries to have better managing process are reviewed. Secondly, the literature for the methodology is evaluated.

\subsection{Literature on Technical Factors}

Pandemics affect most of the countries when they spread all around the world. A country's economic conditions are an important factor for managing these pandemics. In this context, when a country is economically strong, it may be easier to fight pandemic. The main reason for this is that very serious costs occur in the country in this process. For example, healthcare costs increase radically in this process. Additionally, in those times, personnel connections are reduced for fear of infection and social distance is created. As a result, income can reduce since most of the companies have to shut down [22]. So, in the period of epidemics, economically fragile states cannot able to perform the most basic government functions such as offering public services, providing unemployment funds. As a result, it is also clear that pandemics flourish in such environments further decreasing the stability of a functional government. As Maynard and Ong [23] mentioned in their study in developing countries, due to the economic dependency, fragile economies have a negative impact on countries' ability to counteract epidemics.

Technological infrastructure of a country also affects its ability to manage pandemics. The main way to get rid of this type of problem with minimal damage is to find the vaccine to treat the diseases.

Technologically equipped countries are making faster progress in the production of necessary vaccines, as a result of which they are in a better position in combating the pandemic [24]. However, as the vaccine will take time in the process of these rapidly spreading diseases, besides the vaccine, one of the features required to manage the pandemic period well is the adequacy of the country's equipment such as laboratories, assay kits and respirators [25]. Countries with this type of equipment can both detect infected patients beforehand and prevent possible spread, and better control the spread of outbreaks through regular analyzes.

Technological infrastructure that is mentioned above also requires a well-established capacity of health system. Since such diseases spread to many people in a very short time, a country must have a health capacity that can handle this unusual number of patients, adequate number of hospitals, polyclinics and so on [26]. In countries that do not have treatment, it is likely that the system will collapse with a sudden demand for healthcare. In addition, the number of intensive care rooms in hospitals should also be considered, since many of the epidemics from past to present are serious diseases for humans. Apart from physical structures, the number of doctors and health personnel is also critical in the fight against epidemics. In cases where the patient rate is higher than the current healthcare worker rate, delays may occur in the treatments, which delays the control of pandemics [27]. The fact that a country has the power to produce the basic products to be used during the epidemic by its own means also positively 
affects that country's fight against the epidemic. Product imports and exports are mostly minimized because many countries close their borders, especially during times of epidemic [28]. For this reason, it will be very difficult to get medical product without the help from other countries in a pandemic. In this context, countries that can produce their own medical and cleaning materials themselves play a more active role in combating this problem.

\subsection{Literature on Cultural Factors}

Population density can be showed as an important factor to affect the spread of pandemic diseases. In the rapidly globalizing world, cities and metropolitans have replaced village and other settlements. In this case, it caused an increase in population density in certain regions [29]. If there is not an affective population planning, in some regions of the country, the population will be extra dense. Demand for health services is increasing day by day due to pandemics and similar diseases that people may experience as a result of living together [30]. This means an increase in physical areas, health employment and health spending. Increasing the rate of urbanization day by day may cause insufficient health services and epidemic diseases [31]. For this reason, countries that control population density or have properly planned population play a more effective role in preventing the spread of epidemics.

The awareness towards social distance is also an important factor to prevent the spread of the pandemic. In this context, the structure of the cultures of the countries shapes the awareness of the protection of social distance. In high-context cultures, context plays a more decisive role, communication is carried out in ways where indirect, non-verbal communication elements are more dominant [32]. In contrast, in low context cultures, the literal meanings of words are more prominent, communication is more direct. Pandemics will have a higher rate of spread in countries with high-context type of cultural structure, as these cultures often contain more body language and involve people interaction more [33]. Maintaining social distance in high-context will be more difficult in contrast to low-context ones. In lowcontext cultures, people interaction is less than in high-context cultures, thus it makes it easier for such countries to deal with pandemics.

As mentioned above, people's behaviors can be a turning point to prevent or limit epidemics. There can be high concerns that not enough people were following the governments' social-distancing guidance and restrictions as the COVID-19 and other pandemics so that death continued to rise [34]. Besides awareness of people towards social distance, their tendency of obeying rules also contributes limitation of epidemics positively. After a pandemic has started anywhere, many countries have set some restrictions to prevent this problem from spreading. As Tu [35] mentioned that people that obey rules can stand out more easily in comparison to others since they can minimize the contagion effect of epidemic successfully.

Finally, elderly and chronic patient rate to population is also an important factor for countries to manage periods of epidemics. Most types of epidemics and this novel coronavirus has specifically high morbidity in the elderly people than in the general population. This problem created vital problems especially in many European countries which have high rate of elderly people. The main reason behind this issue is 
that they are in more risk compared to others to get pandemic [36]. Besides this, having high chronic patient rate for countries can have a negative impact on the process of pandemics. Both elderly and chronic patients can increase number of infected peoples and even death toll in a country in the period of pandemics, thus it is a crucial threat that countries have to beat [37].

\section{Materials And Methods}

In this study, in general, it was tried to determine which factors countries should pay attention to in the fight against pandemic diseases. In this context, a literature review was conducted on the subject and 8 different criteria were determined. Details of these criteria are given in Table 1.

Table 1. Social and Technical Factors for Struggling Pandemics

\begin{tabular}{ccc}
\hline Dimensions & Criteria & $\begin{array}{c}\text { Supported } \\
\text { Literature }\end{array}$ \\
\hline Technical (Dimension & Economic Conditions (criterion 1) & {$[22],[23]$} \\
1) & Technological Infrastructure (criterion 2) & {$[24],[25]$} \\
& Capacity of health system (criterion 3) & {$[26],[27]$} \\
Social (Dimension 2) & Production Capacity (criterion 4) & {$[28]$} \\
& Population (criterion 5) & {$[29],[30],[31]$} \\
& Social Distance (criterion 6) & {$[32],[33]$} \\
& Tendency of Obeying Rules (criterion 7) & {$[34],[35]$} \\
& Density of elderly people and chronic diseases (criterion & {$[36],[37]$} \\
\hline
\end{tabular}

As can be seen from Table 1, the economic performance of the country is of great importance in order to effectively fight epidemic diseases. In the process of combating pandemic diseases, many different costs will arise. On the other hand, in order to prevent the disease from spreading in this process, the workplaces are closed. This will narrow the country's trade volume. Therefore, countries with high economic performance are more successful in this process. It is obvious that this pandemic caused systemic risks because it affected various industries at the same time. Technological infrastructure is another issue that is important in this process. For the diagnoses to be made effectively and quickly, the country must have equipped laboratories. On the other hand, having sufficient factors such as vaccines and drugs also contributes to the fight against epidemics more successfully. The capacity of the country's health system is also important in this process. In this context, a country with enough hospitals, medical equipment and doctors will be able to solve these problems more easily. In addition to the issues mentioned, the fact that certain health materials can be produced in the country is also very important in this framework. For example, the fact that the needed items such as gloves and masks can be produced in the country will decrease the dependency on the outside in this process.

The social factors of the country also play an important role in combating epidemic diseases. In this context, first, it is necessary to pay attention to the population density in the country. In countries where 
there is no effective population planning, the density of people will be high in certain regions. This will cause epidemic diseases to spread rapidly among people. Social distances between people are very important for these epidemics to be eliminated more easily. Therefore, high context culture countries are having a hard time in this process. On the other hand, another important issue in the fight against epidemics is the compliance of citizens with the state-made boards and recommendations. In this context, the consciousness and compliance level of the people are vital in combating these epidemic diseases. If the recommendations are not implemented by the community, this disease will spread very quickly among people. In addition, epidemics are particularly effective on the elderly and people with chronic diseases. Therefore, if the ratio of this section to the population is very high in a country, it will be much more difficult for this country to fight epidemic diseases.

An analysis will be made with IVIF DEMATEL method in order to determine which of the 8 different criteria is more important. The DEMATEL method is used to determine which of the different criteria affecting a purpose are more effective [38]. The biggest advantage of this method is that the causality relationship between the variables can also be determined [39]. The main reason for this is that it is possible to conduct an effect relationship analysis between factors. Therefore, besides determining which variable is more important, it can be understood which factors affect others more. In the calculation of IVIF DEMATEL approach, evaluations of decision makers are considered. After that, direct relation matrix, normalization matrix and total relation matrix are generated. In the next process, defuzzification process is implemented and the weights of the factors are determined [40]. The details of this calculation process are demonstrated on the Appendix part.

In the evaluation process, 3 decision makers are appointed. These people are experts who study on the epidemiological and social issues as well as economics with at least ten-year experiences. Decision makers consider the linguistic terms for the criteria, dimensions, and alternatives as seen in Table 2.

Table 2. Linguistic Terms for Expert Evaluations

\begin{tabular}{cc}
\hline Linguistic Terms & Percentages \\
\hline Very Low (VL) & 0.1 \\
Low (L) & 0.2 \\
Medium Low (ML) & 0.3 \\
Medium (M) & 0.4 \\
Medium High (MH) & 0.5 \\
High (H) & 0.6 \\
Very High (VH) & 0.7 \\
Absolute (A) & 0.8 \\
\hline
\end{tabular}

\section{Results}


In the first aspect, 3 decision makers made their evaluations by considering the linguistic terms stated in Table 2. This input data is given on the Appendix part (Table A1-A3). After that, direct relation, normalized and total relation matrixes are calculated as in Table A4-A6. In the final stage, the importance levels of the dimensions and criteria are calculated, and the details are summarized in Table 3.

Table 3. Weights of Criteria and Dimensions

\begin{tabular}{ccccc}
\hline Dimensions & Weights & Criteria & $\begin{array}{c}\text { Local } \\
\text { Weights }\end{array}$ & $\begin{array}{c}\text { Global } \\
\text { Weights }\end{array}$ \\
\hline Technical & 0.50 & Economic Conditions (criterion 1) & 0.249 & 0.125 \\
(Dimension 1) & & Technological Infrastructure (criterion 2) & 0.256 & 0.128 \\
& & Capacity of health system (criterion 3) & 0.251 & 0.125 \\
Social (Dimension & 0.50 & Production Capacity (criterion 4) & 0.244 & 0.122 \\
2) & Population (criterion 5) & 0.246 & 0.123 \\
& & Social Distance (criterion 6) & 0.255 & 0.127 \\
& & Tendency of Obeying Rules (criterion 7) & 0.254 & 0.127 \\
& & Density of elderly people and chronic diseases & 0.246 & 0.123 \\
& (criterion 8) & \\
\hline
\end{tabular}

Table 3 indicates that technological infrastructure in the most important factor for the countries to cope with pandemics. In addition to this issue, social distance and tendency of obeying the rules are also essential for the success of the countries to struggle this kind of essential illness. On the other side, it is also concluded that production capacity, population and density of elderly people and chronic diseases play a lower role in this framework in comparison with other ones.

\section{Discussions}

According to the analysis results obtained, it has been determined that the technological infrastructures of countries are the most important factor to be taken in the fight against pandemic diseases. In this context, it is very important to make a rapid and effective diagnosis of the disease in order to control this pandemic in a short time. For this to happen effectively, countries must have the materials in this technical equipment. Therefore, it would be appropriate for countries to invest in the development of this type of technological infrastructure. In addition to this issue, the country's equipped laboratories will contribute to the successful conclusion of this process. Furthermore, for the treatment of this epidemic disease, the country that will be able to develop the relevant vaccine will be more advantageous in this process than others.

In the literature, many different researchers also argued that technological infrastructure of a country has a positive influence on coping with the pandemics. Bernasconi et al. [24] made a study for emerging pandemics. They aimed to understand the ways to stop these problems as soon as possible. In the analysis process, Ebola outbreak in West Africa was considered. They reached a conclusion that the main way to get rid of this type of problem with minimal damage is to find the vaccine to treat the diseases. 
For this purpose, technologically equipped countries are making faster progress in the production of necessary vaccines. Like this study, Chen et al. [25] made an evaluation about coronaviruses. They claimed that countries, which have necessary qualified equipment, can both detect infected patients earlier and prevent possible spread.

Having a social distance will also contribute positively to the countries' struggle with pandemic disease. In this process, the cultures of the people in the country are also important. For example, in a country where warm-blooded people live, it is not easy to achieve social distance between people. This situation makes it difficult for countries to fight the pandemic. Therefore, countries where such people live should be more careful in this process. Parallel to the mentioned issue, the tendency of the people in the country to obey the rules is also an important factor in this framework. For the pandemic not to spread radically, the people in the country must comply with the state-placed quotes and the recommendations made by scientists, without exception. Otherwise, the number of people suffering from the disease will increase rapidly, making it very difficult to combat the epidemic. In this context, it should be explained to people that it is vital for people to follow the rules, especially through role models, through television channels and social media.

This situation was also supported by many different researchers in the literature. For instance, Velavan and Meyer [26] focused on the details of COVID-19 pandemic. They identified that a country should have a health capacity that can handle this unusual number of patients and adequate number of hospitals to prevent these diseases spread to many people in a very short time. Like this study, Schwartz and Graham [27] also made an evaluation on this pandemic. They reached a conclusion that the number of doctors and health personnel is essential for the countries to cope with this pandemic. It is also defined that when the patient rate is higher than the current healthcare worker rate, delays can be occurred in the treatments. This situation has a negative impact on the struggle of this pandemic.

\section{Conclusions}

COVID-19 pandemic caused significant social and economic problems for most of the countries in the world. In the other hand, it is obvious that pandemics lead to systemic risks for these countries. Within this context, pandemics negatively affected companies in different sectors with their contamination effect. As a result, these companies are subject to serious losses. In this study, it is aimed to determine the priority issues that countries should pay attention to while dealing with pandemics. In this context, similar studies in the literature on this subject have been examined and 8 different factors that may be effective in this process have been identified. In the analysis process of the study, there are two different stages. First, an analysis is carried out with the help of IVIF DEMATEL method to understand which of these factors are more important. In the first stage of the analysis, it is determined that technological infrastructure in the most significant issue for the countries to cope with pandemics. Moreover, it is also concluded that social distance and tendency of obeying the rules play an important role to be successful in this situation. Furthermore, economic conditions and capacity of the health system are of medium importance. On the other side, it is also defined that production capacity, population and density of elderly 
people and chronic diseases play a lower role in this framework in comparison with other ones. These results show what the priorities of the countries should be in this process.

In this study, an analysis of pandemic diseases threatening the whole world is carried out. In this context, both priority policies to be implemented in pandemic diseases have been determined and the achievements of countries have been evaluated in the process of combating the COVID-19 pandemic. This illness shows its negative effects in many countries around the world today. Therefore, the analysis results obtained guide these countries towards the policies they need to implement. The most important limitation of this study is to focus only on the strategies that should be applied in the process of fighting the epidemic. An important issue in this process is the development of drugs for these pandemics. It is thought that the academic studies to be carried out for the countries to manage drug production process more effectively will contribute significantly to the literature.

\section{Declarations}

Ethical Approval and Consent to participate: Not applicable

Consent for publication: Not applicable

Availability of data and material: All data analyzed during this study are included in this article.

Competing interests: Not applicable

Funding: Not applicable

Authors' contributions: Shubin Wang made substantial contributions to the conception, design of the work and analysis. Peiqing Yang made substantial contributions to the conception, acquisition and interpretation of data. Serhat Yüksel made substantial contributions to the conception, interpretation of data and analysis. Hasan Dinçer made substantial contributions to the conception and analysis. Gözde Gülseven Ubay made substantial contributions to the interpretation of data and drafted the work or substantively revised it. All authors read and approved the final manuscript.

Acknowledgements: Not applicable

\section{Abbreviations}

DEMATEL: decision making trial and evaluation laboratory

IT2: Interval type-2

IVIF: interval-valued intuitionistic fuzzy

\section{References}


1. Izurieta R. Plagues, Epidemics and Pandemics. In: Global Health Security. Cham: Springer; 2020. pp. 3-11.

2. Cui Y, Wang L, McGoogan JM, Pisani E. Evolution of HIV/AIDS Epidemics in China. In: HIV/AIDS in China. Singapore: Springer; 2020. pp. 3-23.

3. Montibeler EE, de Oliveira DR. Dengue endemic and its impact on the gross national product of Brazilian's economy. Acta Trop. 2018;178:318-26.

4. Novelli M, Burgess LG, Jones A, Ritchie BW. 'No Ebola... still doomed'-The Ebola-induced tourism crisis. Annals of Tourism Research. 2018;70:76-87.

5. Webster F, Rice K, Sud A. A critical content analysis of media reporting on opioids: The social construction of an epidemic. Soc Sci Med. 2020;244:112642.

6. Saunders-Hastings PR, Krewski D. Reviewing the history of pandemic influenza: understanding patterns of emergence and transmission. Pathogens. 2016;5(4):66.

7. Abramowitz SA. Humanitarian morals and money: health sector financing and the prelude to the Liberian Ebola epidemic. Critical African Studies. 2016;8(3):319-34.

8. Zagmutt FJ, Schoenbaum MA, Hill AE. The impact of population, contact, and spatial heterogeneity on epidemic model predictions. Risk Anal. 2016;36(5):939-53.

9. Jafari Y, Othman J. Impact of biofuel development on Malaysian agriculture: A comparative statics, multicommodity, multistage production, partial equilibrium approach. Food Energy Security. 2016;5(3):192-202.

10. Tapia C, Coulton J, Saydam S. Using entropy to assess dynamic behaviour of long-term copper price. Resour Policy. 2020;66:101597.

11. Gul M. A review of occupational health and safety risk assessment approaches based on multicriteria decision-making methods and their fuzzy versions. Human ecological risk assessment: an international journal. 2018;24(7):1723-60.

12. Zhou X, Shi Y, Deng X, Deng Y. D-DEMATEL: A new method to identify critical success factors in emergency management. Saf Sci. 2017;91:93-104.

13. Song W, Zhu Y, Zhao Q. (2020). Analyzing barriers for adopting sustainable online consumption: A rough hierarchical DEMATEL method. Computers \& Industrial Engineering, 106279.

14. Wang LE, Liu HC, Quan MY. Evaluating the risk of failure modes with a hybrid MCDM model under interval-valued intuitionistic fuzzy environments. Comput Ind Eng. 2016;102:175-85.

15. Hoffmann M, Kleine-Weber H, Schroeder S, Krüger N, Herrler T, Erichsen S, ... Müller MA. (2020). SARS-CoV-2 cell entry depends on ACE2 and TMPRSS2 and is blocked by a clinically proven protease inhibitor. Cell.

16. Yang Y, Peng F, Wang R, Guan K, Jiang T, Xu G, ... Chang C. (2020). The deadly coronaviruses: The 2003 SARS pandemic and the 2020 novel coronavirus epidemic in China. Journal of Autoimmunity, 102434. 
17. Phan T. (2020). Genetic diversity and evolution of SARS-CoV-2. Infection, Genetics and Evolution, 104260.

18. Qiu Y, Zhao YB, Wang Q, Li JY, Zhou ZJ, Liao CH, Ge XY. (2020). Predicting the angiotensin converting enzyme 2 (ACE2) utilizing capability as the receptor of SARS-CoV-2. Microbes and Infection.

19. Wilder-Smith A, Chiew CJ, Lee VJ. (2020). Can we contain the COVID-19 outbreak with the same measures as for SARS?. The Lancet Infectious Diseases.

20. Zhang, J. J., Dong, X., Cao, Y. Y., Yuan, Y. D., Yang, Y. B., Yan, Y. Q., ... Gao, Y.D. (2020). Clinical characteristics of 140 patients infected by SARS-CoV-2 in Wuhan,China. Allergy.

21. Miroshnychenko I, Strobl A, Matzler K, De Massis A. (2020). Absorptive capacity, strategic flexibility, and business model innovation: Empirical evidence from Italian SMEs. Journal of Business Research.

22. Botha A, Smulders SA, Combrink HA, Meiring J. (2020). Challenges, barriers and policy development for South African SMMEs-does size matter?. Development Southern Africa, 1-22.

23. Maynard G, Ong C. Economic dependency and HIV/AIDS prevalence in the developing world: a comparative, longitudinal analysis. Sociological Inquiry. 2016;86(2):189-215.

24. Bernasconi, V., Kristiansen, P. A., Whelan, M., Román, R. G., Bettis, A., Yimer, S.A., ... Kumar, A. (2020). Developing vaccines against epidemic-prone emerging infectious diseases. BundesgesundheitsblattGesundheitsforschung-Gesundheitsschutz, 63(1), 65-73.

25. Chen C, Zhou Y, Wang DW. (2020). SARS-CoV-2: a potential novel etiology of fulminant myocarditis. Herz, 1-3.

26. Velavan TP, Meyer CG. The COVID-19 epidemic. Trop Med Int Health. 2020;25(3):278-80.

27. Schwartz DA, Graham AL. Potential maternal and infant outcomes from (Wuhan) coronavirus 2019ncov infecting pregnant women: lessons from SARS, MERS, and other human coronavirus infections. Viruses. 2020;12(2):194.

28. Ghinai, I., McPherson, T. D., Hunter, J. C., Kirking, H. L., Christiansen, D., Joshi,K., ... Fricchione, M. J. (2020). First known person-to-person transmission of severe acute respiratory syndrome coronavirus 2 (SARS-CoV-2) in the USA. The Lancet.

29. Xu B, Tian H, Sabel CE, Xu B. Impacts of Road Traffic Network and Socioeconomic Factors on the Diffusion of 2009 Pandemic Influenza A (H1N1) in Mainland China. Int J Environ Res Public Health. 2019;16(7):1223.

30. Dodds W. Disease Now and Potential Future Pandemics. In: The World's Worst Problems. Cham: Springer; 2019. pp. 31-44.

31. Feng S, Shen C, Xia N, Song W, Fan M, Cowling BJ. (2020). Rational use of face masks in the COVID19 pandemic. The Lancet Respiratory Medicine.

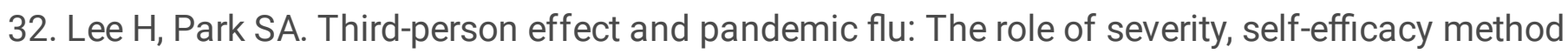
mentions, and message source. Journal of health communication. 2016;21(12):1244-50. 
33. Azizi A, Montalvo C, Espinoza B, Kang Y, Castillo-Chavez C. Epidemics on networks: Reducing disease transmission using health emergency declarations and peer communication. Infectious Disease Modelling. 2020;5:12-22.

34. Kasapoglu A. A Relational Sociological Study on the Effects of Uncertainties in the Case of Influenza in Turkey. International Journal of Clinical Medicine. 2017;8(11):618.

35. Tu J. Conclusion: Moral Experience in a Socialist Neoliberal Polity. In: Health Care Transformation in Contemporary China. Singapore: Springer; 2019. pp. 197-217.

36. Zhang H, Penninger JM, Li Y, Zhong N, Slutsky AS. (2020). Angiotensin-converting enzyme 2 (ACE2) as a SARS-CoV-2 receptor: molecular mechanisms and potential therapeutic target. Intensive Care Medicine, 1-5.

37. Igarashi T, Nihei M, Mizuno J, Inoue T, Kamata M. (2019, November). Subject Selection Bias in Intervention Experiments with Socially Assistive Robots and the Impact on the Representativeness of the Population. In International Conference on Social Robotics (pp. 44-53). Springer, Cham.

38. Karaşan A, Kahraman C. A novel intuitionistic fuzzy DEMATEL-ANP-TOPSIS integrated methodology for freight village location selection. Journal of Intelligent Fuzzy Systems. 2019;36(2):1335-52.

39. Lo HW, Liou JJ, Tzeng GH. Comments on "Sustainable recycling partner selection using fuzzy DEMATEL-AEW-FVIKOR: A case study in small-and-medium enterprises". J Clean Prod. 2019;228:1011-2.

40. Addae BA, Zhang L, Zhou P, Wang F. Analyzing barriers of Smart Energy City in Accra with two-step fuzzy DEMATEL. Cities. 2019;89:218-27.

\section{Supplementary Files}

This is a list of supplementary files associated with this preprint. Click to download.

- SupplementaryMaterial.docx 